\title{
Characterization and scanning probe spectroscopy of radiation-pressure induced mechanical oscillation of a microcavity
}

\author{
T. J. Kippenberg, H. Rokhsari , T. Carmon, K.J. Vahala \\ Department of Applied Physics \\ California Institute of Technology \\ Email: vahala@its.caltech.edu
}

\begin{abstract}
:
(C)2000 Optical Society of America

OCIS codes:, (230.5750) Resonators
\end{abstract}

Microcavities can enter a regime where radiation pressure causes oscillation of mechanical cavity eigenmodes. We present a detailed experimental and theoretical understanding of this effect, and report direct scanning probe spectroscopy of the micro-mechanical modes.

V.B. Braginsky ${ }^{1}$ predicted that due to effects such as radiation pressure the mechanical mirror-eigenmodes of a Fabry-Perot resonator can couple to the optical modes, leading to the regime of parametric oscillation instability. This regime is characterized by regenerative mechanical oscillation of the mechanical cavity eigen-modes. In the past decade, significant theoretical studies have been devoted to this effect in the context of the laser gravitational wave observatory (LIGO) ${ }^{1,2}$, as it limits the achievable sensitivity. We have recently reported for the first time the observation of this nonlinear optical effect in a toroid micro-cavity ${ }^{3}$. Whereas in macroscopic resonators the influence of radiation pressure is weak and only appreciable at high power levels ${ }^{4}$, the mutual coupling of optical and mechanical modes is significantly enhanced in optical microcavities (such as silica microspheres ${ }^{5}$, microdisks or toroids $^{6}$ ) which simultaneously exhibit ultra-high-Q optical modes and high-Q mechanical modes in the RFfrequency range (typically tens of $\mathrm{MHz}$ ). The combination of high optical power and small mechanical mass and dissipation leads to threshold levels in the micro-watt regime for regenerative mechanical oscillations (i.e. parametric oscillation instability).

To understand the mutual coupling of optical and mechanical modes, we have numerically simulated using a finite element solver the micromechanical modes of toroid microcavities. Figure 1 shows strain and stress field of the first three mechanical eigenmodes of a toroid microcavity which are in the RF frequency domain for the cavities used in this work. Within the microcavity, radiation pressure causes a force with radial character ${ }^{1}$. As a result the $n=3$ radial mode, can be efficiently excited as observed by oscillatory behavior in cavity transmission.

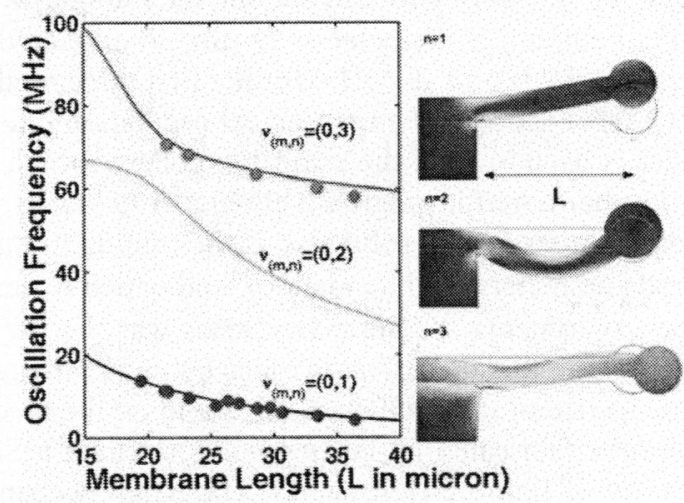

\begin{abstract}
Figure 1: Right panel: Numerical modeling of the micro-mechanical resonances of a silica toroid microcavity using finiteelement-modeling. Shown are the first three resonances. In addition the strain-field is shown (deformation) as well as the stress (color coded). Left panel: The measured resonance frequencies of as a function of membrane length (L). The solid line is the numerically simulated frequency position, based on the exact geometry parameters of the toroid (inferred by SEM). Note that in the experiments, only $n=1$ and $n=3$ modes were observed.
\end{abstract}

\footnotetext{
${ }^{1}$ The momentum transfer occurs, since the modes are confined by continuous total internal reflection, and is a direct consequence of the fact that $\mathrm{CW}$ and $\mathrm{CCW}$ optical trajectories involve a change in linear momentum of $\Delta P=4 h / 2 \pi k n$ per round trip where $\mathrm{n}$ is the number of photons)
} 


\section{QTuK6}

When analyzing this effect in a microcavity using coupled mode theory the threshold power necessary in the tapered fiber waveguide to cause mechanical oscillation is given by:

$$
P_{\text {thresh }}=\frac{\omega_{m}}{\omega_{0}} 2 \pi m_{\text {eff }} R^{2} n_{\text {eff }}\left(\frac{1}{\tau_{m}}\right)\left(\frac{1}{\tau_{0}}\right) \frac{1+K+\left.i 2 \Delta \omega\right|^{2}}{4 K} \tau\left(\frac{1}{1+4 \tau^{2} \Delta \omega_{S}^{2}}-\frac{1}{1+4 \tau^{2} \Delta \omega_{A S}{ }^{2}}\right)^{-1}
$$

In this equation $m_{\text {eff }}$ is the effective mass of the mechanical eigenmode (which is calculated using finite element modeling), $\mathrm{K}$ is the waveguide coupling coefficient ${ }^{7}$, the mechanical quality factor is given by $Q_{m}=\tau_{m} \omega_{m}$ and the optical Q-factor is given by $Q_{0}=\tau_{0} \omega_{0}$. In order to arrive at this equation, we assume that an optical WGM is pumped detuned by $\Delta \omega$ from cavity line-center. It is interesting to note that the threshold for oscillation strongly depends strongly on the optical Q-factor (inverse cubic dependence), while only inverse linearly on the mechanical Q.
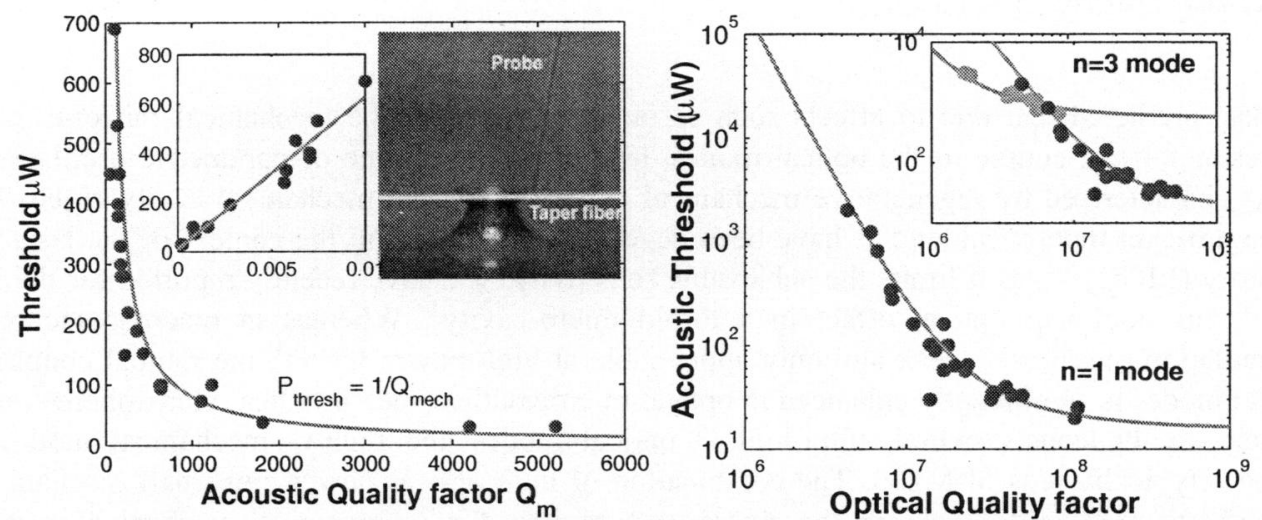

Figure 2: Right panel The mechanical oscillation threshold (launched power in the fiber) as a function of the optical Qfactor. The solid line is a single parameter fit, based on equation 1 . The threshold exhibits an inverse cubic behavior as predicted Left panel: The mechanical threshold (microwatts) versus the mechanical quality factor of the $n=1$ mode. The solid line is the theoretical prediction $(\mathrm{P} \infty 1 / \mathrm{Qm})$. Inset: Optical micrograph from the side of the experimental setup, consisting of a silica-tip probe in contact with a taper-fiber-coupled micro-toroid of 72-micron-principal diameter.

To confirm these theoretical predictions we have investigated the threshold dependence (as given by equation 1) on both optical and mechanical Q-factor. The study was carried out on a single micro-resonator. The micro-toroid under consideration had principal, pillar and toroid diameter of $72 \mu \mathrm{m}, 36 \mu \mathrm{m}$ and $6.8 \mu \mathrm{m}$ and possessed mechanical resonances frequencies at $(4.18 \mathrm{MHz}, 25.6 \mathrm{MHz}$ and $49 \mathrm{MHz})$ for the first three modes $(\mathrm{n}=1,2,3)$.

We indeed observe a strong optical Q dependence of the mechanical oscillation threshold as shown in the right panel of figure 2. A double logarithmic plot is used to infer the critical exponent, which shows excellent agreement with the predicted $\mathrm{Q}$ dependence $\left(1 / \mathrm{Q}^{3}\right)$. Furthermore for progressively higher $\mathrm{Q}$ values, a roll-over is observed, which is in agreement with the predictions of equation 1. The solid line is the minimum threshold as given by equation (1) based on the experimentally measured parameters $\left(\mathrm{R}, \mathrm{Q}_{\mathrm{m}}, \omega_{\mathrm{m}}, \omega_{0}\right)$, and which is minimized with respect to detuning $(\Delta \omega)$ and coupling $(\mathrm{K})$ to reflect the experiments. The effective mass was the only fit parameter used. As evident very good agreement is obtained, confirming that this effect is cause by radiation pressure.

We next proceeded to verify the dependence of the oscillation threshold on the mechanical Quality factor $\left(\mathrm{Q}_{\mathrm{m}}\right)$. We achieved this by using a silica-nanoprobe fabricated by heating and stretching a SMF fiber using a $\mathrm{CO} 2$ laser. An optical micrograph is shown in the inset of figure 2 (left panel). The nano-mechanical probe was mounted on a 3axis piezoelectric stack, and positioned to the taper-fiber coupled microtoroid. When the nanoprobe was brought into contact with parts of the resonator exhibiting high-amplitude mechanical oscillations (compare figure 1) a reduction of the mechanical Q-factor was observed, while leaving the optical Q-factor unaffected. Without contact the mechanical $\mathrm{Q}$ was measured to be $\sim 5000$ for the $\mathrm{n}=1$ mode, and upon progressive increase in tip pressure (controlled via piezo-electric distance) the mechanical $\mathrm{Q}$ could be continuously decreased by nearly two orders of magnitude, down to a value of 50. For each mechanical $Q$ the minimum threshold was measured for the $n=1$ flexural mode. Figure 2 (left panel) shows the measured minimum threshold for the mechanical oscillations as a function of 


\section{QTuK6}

mechanical Q-factor, clearly revealing the classical 1/Qm oscillator dependence of the oscillation threshold, in agreement with equation 1 , as well as theoretical predictions of Braginsky ${ }^{5,8}$.

We also used the nanoprobe to directly spatially image the micromechanical modes, by measuring the spatial dependence of the induced change in mechanical $Q$ and frequency. We have successful implemented this method and performed a radial scan using the nano-probe along the silica disk. Figure 5 shows the measured frequency shift vs. probe position for the first and second order mode. The measured data show remarkably good agreement with the numerically calculated mode distribution, as shown in the inset of figure 3 and thus allow direct imaging of the micro-mechanical modes of the toroid. This method should also be applicable to other nano- and micro-mechanical systems ${ }^{9}$ and is to our knowledge the first demonstration of scanning probe microscopy of micro-mechanical modes.
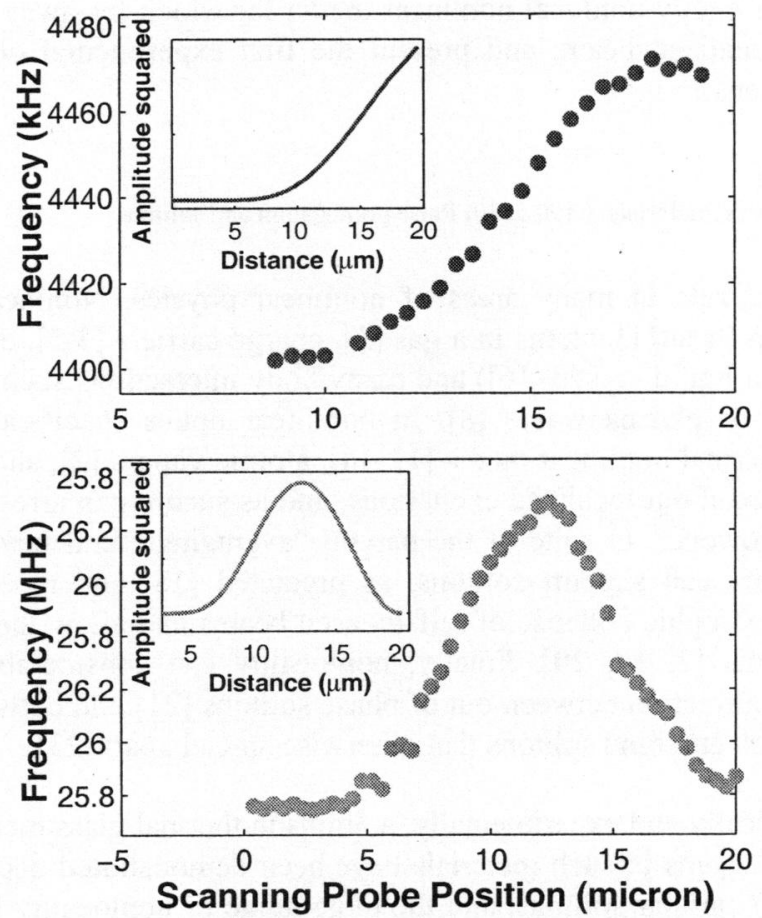

Figure 3: Scanning probe microscopy of the micro-mechanical resonances using the toroid microcavity from the previous graphs. In the experiment the scanning probe position corresponds to the radial position of the silica nano-probe. The dotted line indicates the displacement inferred by measurement of the resonant frequency. Upper graph: $\mathrm{n}=1$ (fundamental) flexural mode $(\mathrm{v}=4.4 \mathrm{MHz})$. Lower graph: $\mathrm{n}=2$ second order mode $(\mathrm{v}=25.8 \mathrm{MHz})$. Insets: The numerically calculated $\mathrm{z}$-component of the amplitude of the mechanical eigenmodes.

1. Braginsky, V. B., Strigin, S. E. \& Vyatchanin, S. P. Parametric oscillatory instability in Fabry-Perot interferometer. Physics Letters A 287, 331-338 (2001).

2. Kells, W. \& D'Ambrosio, E. Considerations of parametric instability in Fabry-Perot interferometer. Physics Letters A 229, 326-330 (2002).

3. Rokhsari, H., Kippenberg, T. J., Carmon, T. \& Vahala, K. J. Photonic quartz oscillator. (in review) (2004).

4. Dorsel, A., McCullen, J. D., Meystre, P., Vignes, E. \& Walther, H. Optical Bistability and Mirror Confinement Induced by Radiation Pressure. Physical Review Letters 51, 1550-1553 (1983).

5. Braginsky, V. B., Gorodetsky, M. L. \& Ilchenko, V. S. Quality-Factor and Nonlinear Properties of Optical Whispering-Gallery Modes. Physics Letters A 137, 393-397 (1989).

6. Armani, D. K., Kippenberg, T. J., Spillane, S. M. \& Vahala, K. J. Ultra-high-Q toroid microcavity on a chip. Nature 421, $925-928$ (2003).

7. Kippenberg, T. J., Spillane, S. M. \& Vahala, K. J. Modal coupling in traveling-wave resonators. Optics Letters 27, 1669-1671 (2002)

8. Braginsky, V. B., Gorodetsky, M. L., Ilchenko, V. S. \& Vyatchanin, S. P. On the Ultimate Sensitivity in Coordinate Measurements. Physics Letters A 179, 244-248 (1993).

9. Craighead, H. G. Nanoelectromechanical systems. Science 290, 1532-1535 (2000). 